\title{
1 Article
}

2 Moving Source Depth Estimation Using a Horizontal Line 3 Array in Shallow Water Waveguides \\ Guolong Liang ${ }^{1,2,3}$, Yifeng Zhang ${ }^{1,2,3}$, Nan Zou ${ }^{1,2,3, *}$ and Jinjin Wang ${ }^{1,2,3}$ \\ 1 Acoustic Science and Technology Laboratory, Harbin Engineering University, Harbin, China; \\ 2 Key Laboratory of Marine Information Acquisition and Security (Harbin Engineering University), Ministry \\ of Industry and Information Technology; Harbin 150001, China \\ 3 College of Underwater Acoustic Engineering, Harbin Engineering University, Harbin, China; \\ liangguolong@hrbeu.edu.cn; 1477989475@qq.com \\ * Correspondence: zounan@hrbeu.edu.cn; Tel.: +86-151-2450-3518
}

Abstract: In this study, a matched-mode autoregressive source depth estimation method (MMAR) based on autoregressive (AR) wavenumber estimation is proposed for a moving source in shallow water waveguides. The signal original frequency and the environmental parameters, namely, the sound speed profile and bottom properties are known as a prior knowledge. The mode wavenumbers are estimated by the AR modal wavenumber spectrum. On the basis of the mode wavenumber estimation, the mode amplitudes can be estimated by the wavenumber spectrum that is obtained by generalized Hankel transform. The source depth estimation is determined by the peak of source depth function wherein the data mode best matches the replica mode that is calculated using a propagation model. Compared with other methods of moving source depth estimation, the proposed method exhibits a better performance in source depth estimation under low signal-to-noise ratio or the small range span. The selection of horizontal line array depth is illustrated by simulation and normal mode theory in details.

Keywords: modal wavenumber spectrum; match mode; horizontal line array; moving source

\section{Introduction}

Matched field processing (MFP) [1-2] or the equivalent matched mode processing (MMP) [3] is

a popular method for localizing the source in complex propagation environments. Conventional MFP obtains the range depth function, that is, the correlation between the replica and the measured fields. The source location is determined by the peak of the range depth function. MFP can localize the source accurately in a range-independent or slowly varying environment without considering any environmental mismatches. When the sound propagation is not exactly modeled, the range and depth estimated by MFP generally present an error, which is referred to as the environmental mismatch problem [4]. The need for depth estimation is more pressing than range estimation for some applications. The source range and depth are estimated simultaneously by MFP, which indicates that depth estimation depends on range estimation. The robustness of depth estimation decreases because of the sensitivity of range estimation to sound speed profile (SSP). MFP always requires a vertical line array (VLA) or a horizontal line array (HLA) with large aperture. However, real application of large aperture array is difficult because of the installation platform. The application of MFP is restricted by the aperture problem and the environmental mismatch problem. Consequently, an active area of research in recent years has been devoted to estimate source depth robustly by a single hydrophone or a short line array [5-7].

A class of methods that has shown some promise in passive location exploits the properties of the reliable acoustic path (RAP) [8]. Such methods reduce the requirement of array aperture. The difficulty associated with the propagation of RAP limits the application of the method in shallow water. Depth estimation for shallow water waveguides is generally performed in two steps: mode filtering first and then MMP [3]. The modal amplitudes are determined by mode filters, and then the MMP is used to estimate the source location on basis of the modal amplitudes. However, the mode 
filters always require well-sampling of the water column by the VLA; otherwise, the mode filtering will become an ill-posed problem. The difficulty in source depth estimation using a short line array is the lack of spatial information. Thus, some previous studies have relied on the multi-frequency characteristics of wideband signals in estimating source depth [9-10]. Considering the dispersion phenomenon of signals in shallow water, some scholars have estimated source depth on the basis of the related information of marine environment contained in dispersion [11]. However, the above mentioned studies have restricted the signal form to be broadband and few works on source depth estimation for signal with low-frequency line spectrum are available. Recently, data-based MFP/MMP is being proposed for a moving source by using a full-spanning VLA, which is free of the environmental mismatch problem in theory [12-13]. Yang [7] proposed a method based on the idea of data-based MFP/MMP to estimate moving source depth, and this method requires only a single hydrophone. It is more robust than MFP for depth estimation because of not requiring source range estimation. However, the method presents some inherent drawbacks. (1) The method requires source travelling a sufficient range in radial direction owing to that the mode wavenumbers are estimated by synthetic aperture modal beamforming $(\mathrm{SAB})$. Some of the horizontal wavenumbers cannot be estimated, when the moving range does not meet the requirement. Source depth estimation based on synthetic aperture beaming may be incorrect. (2) The model assumes that the signal possesses high signal-to-noise (SNR). Such requirement of SNR is difficult to meet in passive positioning. (3) The algorithm is difficult to apply when the source is not moving in the radial direction.

In this study, we propose a source depth estimation approach with an HLA for a moving source in a range-independent environment, without knowing source absolute range information. When beam steers to the source direction, the signal is enhanced after beamforming. We apply the autoregressive (AR) model to the signal enhanced by array gain to estimate the wavenumbers. The AR wavenumber spectrum is unsuitable to estimate mode amplitudes. Thus, the generalized/modified Hankel transform is applied to the data. The source depth is estimated by the peak of the depth ambiguity function wherein the mode depth functions best match the mode amplitudes. Compared with $\mathrm{SAB}$, the proposed method effectively improves the depth estimation performance for the data with short range span or low SNR, and exhibits better adaptability to the notion of the observation platform. In addition, the proposed method can be applied to underwater unmanned vehicles or the autonomous underwater vehicles because it identifies the peaks of wavenumber spectrum automatically.

The remainder of the paper is organized as follows. The wavenumber estimation for the beamforming output is developed in Section 2. In Section 2.1, the generalized Hankel transform is extended to beamforming output in a range independent environment [7]. A method for wavenumber estimation based on an AR model without knowing the source range is proposed in Section 2.2. Source depth estimation based on wavenumber spectrum is introduced in Sec 3 . The factors that influence the performance of the algorithm are explored in Section 4 . The performance of the algorithm on the data with different SNRs and different range spans is analyzed in Section 4.1. Given that the HLA depth also affects the performance of the algorithm, the effect of the laying depth of HLA on the algorithm and its selection method are examined in details in Section 4.2. A summary and discussion is provided in Section 5 .

\section{Mode Wavenumber Estimation}

We assume that source travels at a fixed depth during observation in a range-independent environment. The considered HLA possesses $N$ receivers that are uniformly spaced with $d$. The first element of HLA is the reference. The far-field source radiates the continuous wave signal, with a look direction $\theta_{i} . \theta_{i}$ is the true value of the look direction at the $i$ th moment. The range between source and reference array element is $r_{i}$ at the $i$ th moment. In shallow water waveguides, we suppress a time dependence of the form $e^{j \omega t}$ with $\omega$ positive, thus, the pressure field of the $n$th element at the $i$ th moment can be represented using normal mode theory as 


$$
p_{n}\left(r_{i}, z_{r}\right)=\sqrt{2 \pi} e^{-j \frac{\pi}{4}} \sum_{m=1}^{M} \phi_{m}\left(z_{s}\right) \phi_{m}\left(z_{r}\right) \frac{\exp \left\{-j\left(k_{m}-j \alpha_{m}\right)\left[r_{i}+(n-1) d \sin \theta_{i}\right]\right\}}{\sqrt{k_{m}\left[r_{i}+(n-1) d \sin \theta_{i}\right]}},
$$

where $k_{m}, \alpha_{m}$, and $\phi_{m}$ are the wavenumber, the attenuation coefficient, and the mode depth function, respectively, which all belong to the $m$ th mode; $z_{s}$ and $z_{r}$ are the source and HLA depths, respectively; and $M$ is the number of modes considered in the propagation model.

The source is at the far field, such that $r_{i}+(n-1) d \sin \theta \approx r_{i}$. Equation. (1) is simplified as

$$
\begin{aligned}
p_{n}\left(r_{i}, z_{r}\right) & =\sqrt{2 \pi} e^{-j \frac{\pi}{4}} \sum_{m=1}^{M} \phi_{m}\left(z_{s}\right) \phi_{m}\left(z_{r}\right) \frac{\exp \left\{-j\left(k_{m}-j \alpha_{m}\right)\left[r_{i}+(n-1) d \sin \theta_{i}\right]\right\}}{\sqrt{k_{m} r_{i}}}, \\
& =\sum_{m=1}^{M} A_{m} \exp \left\{-j\left(k_{m}-j \alpha_{m}\right)\left[r_{i}+(n-1) d \sin \theta_{i}\right]\right\}
\end{aligned}
$$

where $A_{m}=\sqrt{2 \pi} e^{-j \frac{\pi}{4}} \frac{\phi_{m}\left(z_{s}\right) \phi_{m}\left(z_{r}\right)}{\sqrt{k_{m} r_{i}}}$. To improve the SNR, beamforming is performed on the signal received on the HLA at the $i$ th moment.

$$
B\left(\hat{\theta}_{i}\right)=\frac{1}{N} \sum_{n=1}^{N} w_{n}\left(\hat{\theta}_{i}\right)\left(p_{n}\left(r_{i}, z_{r}\right)+n_{n}\left(r_{i}, z_{r}\right)\right),
$$

$n_{n}\left(r_{i}, z_{r}\right)$ is modeled as the Gaussian white noise. For conventional beamforming, the output of beamforming is written as

$$
B\left(\hat{\theta}_{i}\right)=\frac{1}{N} \sum_{n=1}^{N} e^{j k(n-1) d \sin \hat{\theta}_{i}}\left(p_{n}\left(r_{i}, z_{r}\right)+n_{n}\left(r_{i}, z_{r}\right)\right),
$$

After substituting Equation. (2) into Equation. (4), while ignoring noise, we obtain

$$
B\left(\hat{\theta}_{i}\right)=\frac{1}{N} \sum_{m=1}^{M} A_{m} e^{-j k_{m} r_{i}-\alpha_{m} r_{i}} \operatorname{sinb}\left(X_{m}\right),
$$

where $X_{m}=\left(-k_{m}-j \alpha_{m}\right) \sin \theta_{i}+k \sin \hat{\theta}_{i}, \quad \operatorname{sinb}\left(X_{m}\right)=\sin \left(\frac{N d}{2} X_{m}\right) / \sin \left(\frac{d}{2} X_{m}\right) . \hat{\theta}_{i}$ is the estimation of the look direction, which can be obtained by many methods [14]. In order to explain the algorithm conveniently, we discuss the method of estimating the sound source depth when source moves away from the HLA under the assumption that $\hat{\theta}_{i}=\theta_{i}=\theta$.

\subsection{Wavenumber estimation using generalized Hankel transform}

In range-independent shallow water waveguides, a Hankel transform pair presents the relationship between the complex pressure field $p\left(r ; z_{s}, z_{r}\right)$ and the Green's function $g\left(k_{r} ; z_{s}, z_{r}\right)$ [15]

$$
\begin{aligned}
& p\left(r ; z_{s}, z_{r}\right)=\int_{0}^{+\infty} g\left(k_{r} ; z_{s}, z_{r}\right) J_{0}\left(k_{r} r\right) k_{r} d k_{r}, \\
& g\left(k_{r} ; z_{s}, z_{r}\right)=\int_{0}^{+\infty} p\left(r ; z_{s}, z_{r}\right) J_{0}\left(k_{r} r\right) r d r
\end{aligned}
$$

where $J_{0}$ is the zeroth-order Bessel function.

Considering the computation of Hankel transform, the Green's function is often approximated by an inverse Fourier transform (FT) as [15] 


$$
g\left(k_{r} ; z_{s}, z_{r}\right) \sim \frac{e^{i \frac{\pi}{4}}}{\sqrt{2 \pi k_{r}}} \int_{-\infty}^{+\infty} p\left(r ; z_{s}, z_{r}\right) e^{i k_{r} r} \sqrt{r} d r, \quad k_{r} r \gg 1,
$$

where

$$
a_{m}=\frac{e^{j\left[\left(k_{r}-k_{m}\right)-\alpha_{m}\right]\left(r_{0}+R\right)}-e^{j\left[\left(k_{r}-k_{m}\right)-\alpha_{m}\right] r_{0}}}{j \sqrt{k_{r} k_{m}}} \sin b\left(X_{m}\right),
$$

133 When $k_{r}=k_{m}$, the value of the $m$ th item is much larger than the others in Equation. (10).Thus, 134 the wavenumber spectral peak at $k_{r}=k_{m}$ is given by

$$
\begin{gathered}
\bar{g}\left(k_{m}, z_{r}\right) \sim b_{m} \phi_{m}\left(z_{r}\right), \\
b_{m}=\frac{2 e^{-\alpha_{0} r^{\prime}}}{\alpha_{m} k_{m}} \sinh \left(\frac{\alpha_{m} R}{2}\right) \phi_{m}\left(z_{s}\right) \sin b\left(X_{m}\right),
\end{gathered}
$$

136 where $r^{\prime}=r_{0}+\frac{R}{2}$. This expression can be described by the matrix form. 


$$
\boldsymbol{g}=\boldsymbol{\Phi} \cdot \boldsymbol{b}
$$

137 where

$$
\begin{gathered}
\boldsymbol{g}=\left[\bar{g}\left(k_{1}, z_{r}\right), \bar{g}\left(k_{2}, z_{r}\right), \cdots, \bar{g}\left(k_{M}, z_{r}\right)\right]^{T}, \\
\boldsymbol{\Phi}=\operatorname{diag}\left(\left[\phi_{1}\left(z_{r}\right), \phi_{2}\left(z_{r}\right), \cdots, \phi_{M}\left(z_{r}\right)\right]\right), \\
\boldsymbol{b}=\left[b_{1}, b_{2}, \cdots, b_{M}\right]^{T} .
\end{gathered}
$$

138

139

140

141

142

143

144

145

146

147

148

149

150

151

152

153

154

155

156

157

158

159

160

The aforementioned wavenumber estimation method is based on the FT, and is convenient to be calculated by FFT. However, the FT-based approach presets some inherent disadvantages: (1) The spectral resolution of FT is limited by the range span. To resolve the modal wavenumbers between the $i$ th and $j$ th modes, the range needs to be larger than the interference distance $d_{i j}=2 \pi /\left|k_{i}-k_{j}\right|$. If all the wavenumbers are estimated, then the range must be larger than all interference distances. (2) The spectrum leakage occurs seriously when the method is applied to the data with a short range span. The sidelobes of strong spectral components can contaminate the weak spectral components or generate a false spectral peak. With the increase in source frequency or waveguide depth, the lower mode wavenumbers will closely group together. As a result, the wavenumbers are difficult to be estimated in this environment. Considering the drawbacks of FT, the modern spectral estimation methods can be applied to wavenumber estimation.

\subsection{Wavenumber estimation using AR model}

Several methods have been developed to extract the mode wavenumbers, such as Prony's method, the signal subspace algorithms and matrix-pencil $[16,17]$. However, these methods regard the number of wavenumbers as a priori. The number of wavenumbers cannot be known correctly in practice. The AR spectral estimators based on an all-pole model are often used to extract the spectral peaks in frequency estimation [17]. The AR estimator does not need to know the number of wavenumbers, and is thus attractive to estimate wavenumbers. However, the source range must be known for the AR estimator. Consequently, improved AR estimator should be used to extract the mode wavenumbers.

The method can be divided into three steps. First, data are preprocessed by

$$
y[i]=B\left(r_{i}\right) S\left(r_{i}\right) \quad i=1,2 \ldots L,
$$

Second, considering that $y[i]$ can be described as the output of a linear system, we construct the AR model as

$$
y[i]=-\sum_{k=1}^{p} a[k] y[i-k]+u[i],
$$

161 where $p$ is the order of the AR model to represent the data, and is often set to $\frac{2 N}{3}$ [17]. Finally,

162 we assume that $u[i]$ is a zero mean white noise sequence with $\sigma^{2}$. Thus, the wavenumber spectral 163 density $P_{A R}$ is expressed as:

$$
P_{A R}(l)=\frac{\sigma^{2}}{\left|1+\sum_{k=1}^{p} a[k] \exp [-i l k]\right|}
$$


$164 P_{A R}$ is obtained by estimating the coefficients $a[1], a[2], \cdots, a[p]$ and $\sigma^{2}$. The

165

166

167

168

169

170

171

172

173

174

175

176

177

178

179

180

above-mentioned coefficients can be estimated in different ways. We select the modified covariance approach because it avoids the spectral line splitting effectively [18]. The locations of peaks in $P_{A R}$ yield the wavenumbers estimated by AR model. The peak levels estimated by AR estimator possess large variances [18]. Thus, AR estimator is unsuitable to estimate the modal amplitudes.

\section{Matched-Mode Source Depth Estimation}

As discussed above, the wavenumbers can be estimated by AR spectrum. However, AR spectrum is unsuitable to estimate the modal amplitudes. The performance of estimating wavenumbers degrades by using the generalized Hankel transform. The performance is effected by false spectral peaks and spectral resolution. To combine the advantages of two methods, the modal amplitudes are estimated by generalized Hankel transform with a prior knowledge of wavenumbers that are estimated by AR model. Thus, we estimate the wavenumber $k_{m}$ by AR model, and then obtain $\bar{g}\left(k_{m}, z_{r}\right)$ in the wavenumber spectrum on basis of the generalized Hankel transform.

According Equation. (14), we use a source depth ambiguity function to estimate source depth, and this function is expressed as

$$
D(z)=\varphi(z) b \boldsymbol{b} \varphi^{H}(z)
$$

where

$$
\varphi(z)=\left[\phi_{1}(z), \phi_{2}(z), \cdots, \phi_{M}(z)\right]
$$

$\boldsymbol{b}$ can be solved by

$$
\begin{gathered}
\boldsymbol{b}=(\boldsymbol{\Phi}+\boldsymbol{U})^{-1} \boldsymbol{g} \\
\boldsymbol{U}=\operatorname{diag}\left(\Delta / \phi_{1}\left(z_{r}\right), \Delta / \phi_{2}\left(z_{r}\right), \cdots, \Delta / \phi_{M}\left(z_{r}\right)\right),
\end{gathered}
$$

181 where $\Delta$ is a small amount (on the order of one half of the maximum value of the mode function) 182 for preventing the singularity of $\boldsymbol{\Phi}$. Wavenumbers $k_{m}^{\prime}$ and $\phi_{m}(z)$ are calculated by KRAKEN on 183 the basis of a given frequency and environmental information (SSP and bottom properties). Some 184 modes cannot be resolved even if we use the AR spectrum, such that the number of $\bar{g}\left(k_{m}, z_{r}\right)$ is 185 less than $M$. We assume that only $M_{0}$ order is estimated. $\phi_{m}(z)$ with the same order of $186 \bar{g}\left(k_{m}, z_{r}\right)$ can be determined by solving the problem as follows

$$
\left\{\begin{array}{l}
\min \left(\boldsymbol{k}-\boldsymbol{k}_{0}\right)^{\boldsymbol{H}}\left(\boldsymbol{k}-\boldsymbol{k}_{0}\right) \\
\text { s.t. } \quad \boldsymbol{k}_{0}(1)<\boldsymbol{k}_{0}(2)<\cdots<\boldsymbol{k}_{0}\left(M_{0}\right)^{\prime}
\end{array}\right.
$$

187 where

$$
\begin{gathered}
\boldsymbol{k}=\left[k_{1}, k_{2}, \cdots, k_{M_{0}}\right]^{T}, \\
\boldsymbol{k}^{\prime}=\left[k_{1}^{\prime}, k_{2}^{\prime} \cdots, k_{M}^{\prime}\right]^{T}, \\
\boldsymbol{k}_{0} \subseteq \boldsymbol{k}^{\prime} .
\end{gathered}
$$

$\boldsymbol{k}$ is an $M_{0} \times 1$ vector, $\boldsymbol{k}^{\prime}$ is an $M \times 1$ vector, and $\boldsymbol{k}_{0}$ is an $M_{0} \times 1$ vector. 
This section presents comparisons of the source depth estimation results of the SAB and the proposed matched-mode autoregressive source depth estimation method (MMAR). Different range span and SNR should be considered in evaluating the performance of SAB and MMAR in estimating source depth. For $\mathrm{SAB}$, source depth can be estimated using only a single hydrophone under the assumption that source moves away from receiver at a constant speed during the observation time. For convenient comparison, we assume the source of SAB moves along the beam direction of HLA. Apart from the influence of SNR and range span on the performance of the algorithms, the influence of the HLA depth on MMAR is also considered. We study this effect from theory and simulation.

The pressure field is generated using the KRAKEN program. The SSP for simulation is shown in Figure 1, and the bottom properties are referred to Reference 7. The consider a HLA possesses $N$ receivers that are uniformly spaced with $d$, where $d=\frac{\lambda}{2}$. In studying the difference in depth estimation performance, we consider two sources depths of 4 and $50 \mathrm{~m}$, which correspond to shallow and deep sources, respectively. The initial source range is $5010 \mathrm{~m}$, but this information is assumed to be unknown. The performance influenced by the unknown initial source range has been discussed in Ref. 7. We assume that each source moves away from the HLA with a speed of $2.5 \mathrm{~m} / \mathrm{s}$, and radiates the narrowband signal with $350 \mathrm{~Hz}$. The SNR in this simulation is defined as:

$$
S N R=\left.10 \lg \frac{P_{s}}{P_{n}}\right|_{r=r_{0}} .
$$

According to Equation. (29), when source range is $5010 \mathrm{~m}$, the SNR is described by the ratio of signal power and noise power at receiver. The array gain is already considered in SNR.

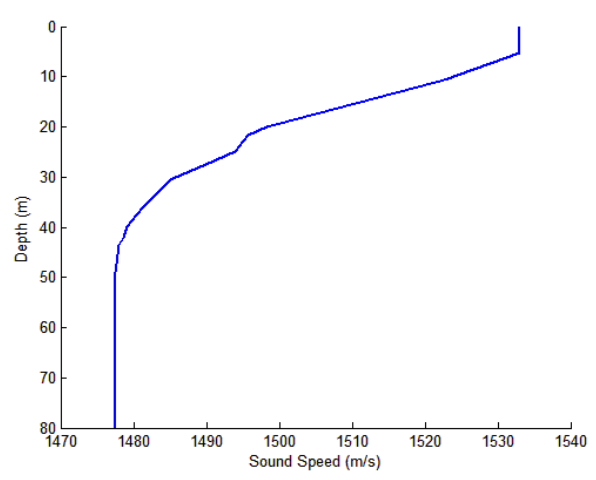

Figure 1. Sound speed profile used in this experiment, which is typical in shallow waveguides.

\subsection{Comparison of $S A B$ and $M M A R$ applied to simulation data}

The HLA depth is $70 \mathrm{~m}$ without special instructions. The effectiveness of our algorithm is analyzed in the following three cases and compared with that of SAB. A unified description is given to avoid duplication, that is, the deep source is expressed by the blue line and the shallow source is expressed by the red line. In Figure 2-4, subplot (a) shows the wavenumber spectrum obtained by the generalized Hankel transform; subplot (b) shows the AR wavenumber spectrum obtained by AR model, which is used to estimate the mode wavenumbers in MMAR; the source depths estimated using SAB and MMAR are shown in subplot (c) and (d). We determine the performance of source depth estimation under three cases.

1. Range span is sufficient and SNR is high

The source moves $4990 \mathrm{~m}$ during the observation time. The SNR is $40 \mathrm{~dB}$. From Figure $2 \mathrm{a}, \mathrm{b}$, the peaks of wavenumber spectrum obtained by two methods are found to be clear. Therefore, we can estimate wavenumbers accurately using the two methods under this simulation condition. Both methods yield good depth estimation results as shown in Figure 2c,d. Comparing Figure 2c,d shows that the sidelobes of the depth estimation in Figure $2 d$ are smaller than those in Figure $2 c$, especially for the shallow source. The number of mode wavenumbers estimated is 9 in Figure $2 \mathrm{a}$ and the 
number is 11 in Figure $2 \mathrm{~b}$. The more mode wavenumbers are estimated correctly, the more information can be taken into depth estimation. The performance of depth estimation can be improved by increasing the number of mode wavenumbers estimated. Small sidelobes can also be obtained. Thus, when the range span is sufficient and the SNR is high, the performance of depth estimation using MMAR is slightly better than the performance of that using SAB.

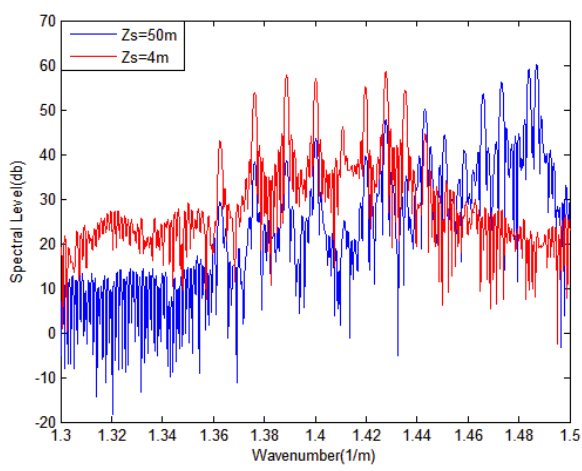

(a)

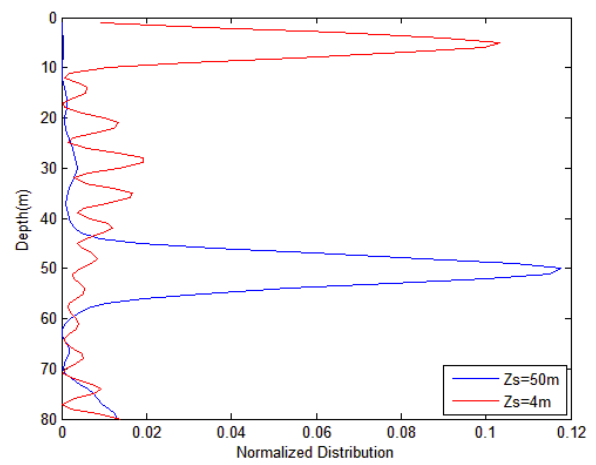

(c)

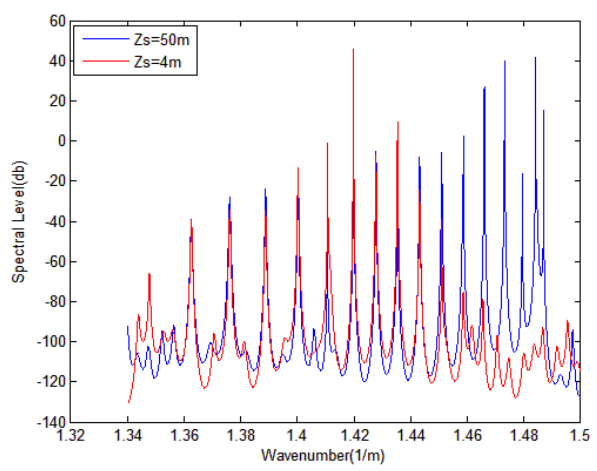

(b)

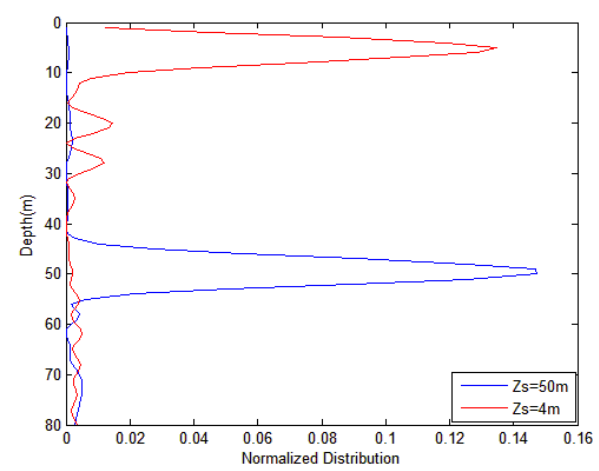

(d)

Figure 2. Comparison of $\mathrm{SAB}$ and MMAR in terms of source depth estimation by using simulated pressure field data covering the range span of $4990 \mathrm{~m}$ with an SNR of $40 \mathrm{~dB}$. (a) Mode wavenumber extracted using the modified Hankel transform. (b) Wavenumber estimated using the AR model. (c) Depth estimation using SAB. (d) Depth estimation using MMAR.

2. Range span is sufficient and SNR is low

In this simulation, the range span is $4990 \mathrm{~m}$ and the SNR is $5 \mathrm{~dB}$. Only the deep source can be estimated correctly as shown in Figure 3c. By contrast, the results in Figure $3 d$ show good depth estimation for the shallow and deep sources. SAB presents difficulty in estimating the mode wavenumbers correctly as shown in Figure 3a. This difficulty is due to the influence of sidelobe mask. MMAR based on AR model can reduce the influence of sidelobe mask. Thus, the spectral peaks can be determined and the mode wavenumbers can be estimated accurately. When the range span is sufficient and the SNR is low, the performance of depth estimation using MMAR improves greatly compared with the performance of that using SAB.
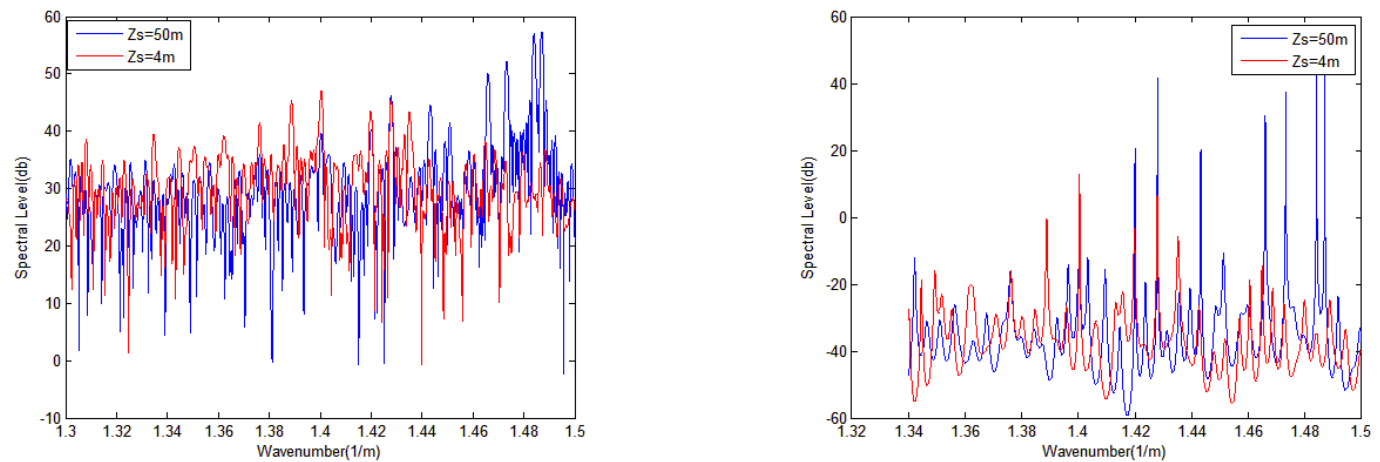
(a)

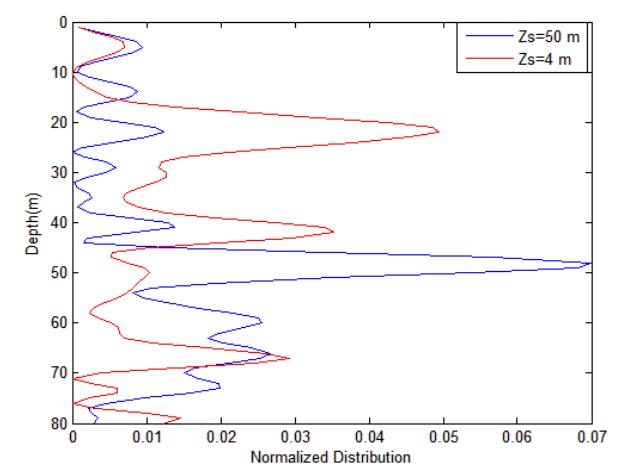

(c) (b)

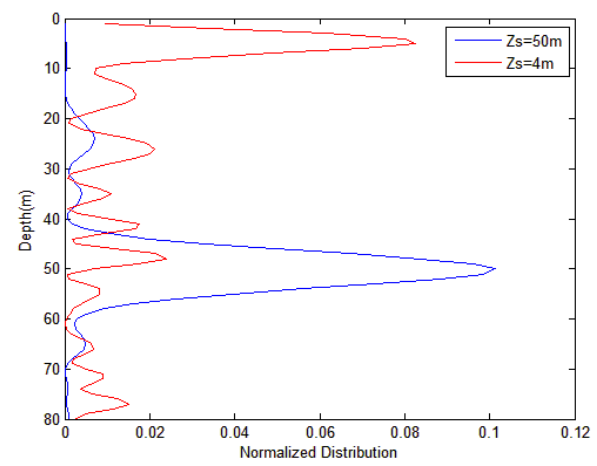

(d)

Figure 3. Comparison of SAB and MMAR in terms of source depth estimation by using simulated pressure field data covering the range span of $4990 \mathrm{~m}$ with an SNR of $5 \mathrm{~dB}$. (a) Mode wavenumber extracted using the modified Hankel transform of SAB. (b) Wavenumber estimated by the AR model

3. Range span is insufficient and SNR is high

The source moves $1990 \mathrm{~m}$ and the SNR is $40 \mathrm{~dB}$ in this simulation. Both methods can estimate the shallow source depth effectively under this condition as shown in Figure 4c,d. SAB fails whereas MMAR succeeds to estimate deep source depth. SAB mistakenly selects the sidelobe peaks as estimations of the mode wavenumbers, and the false wavenumbers are shown in Figure 4a. By contrast, the peaks of the main lobe can be extracted effectively and the miscarriage of justice can be reduced using AR model. When the range span is insufficient and the SNR is high, the performance of MMAR is better than the SAB. In particular, MMAR performs obviously better than SAB in terms of estimation of deep source depth.

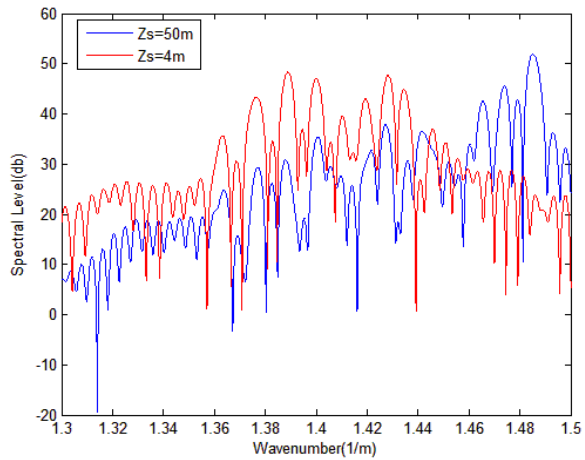

(a)

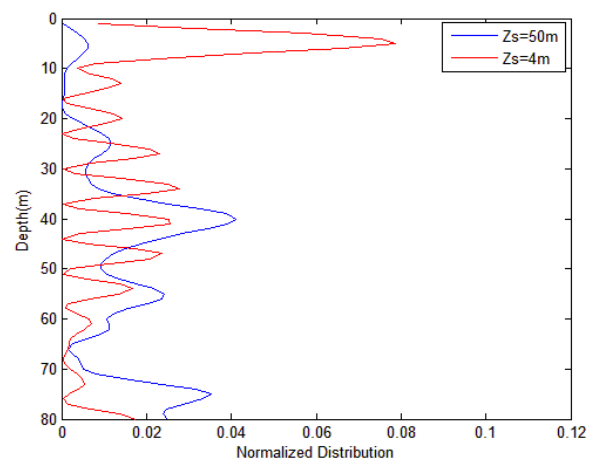

(c)

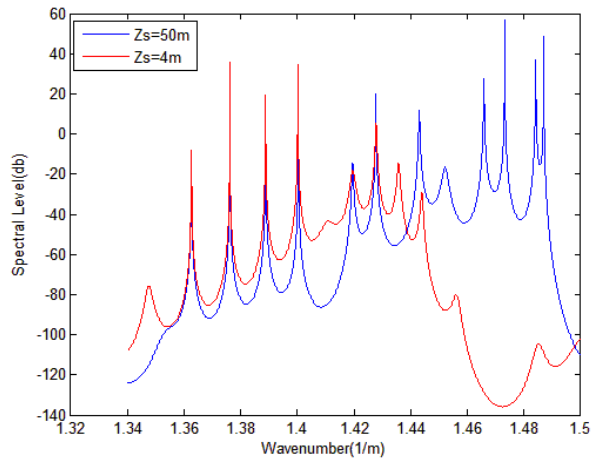

(b)

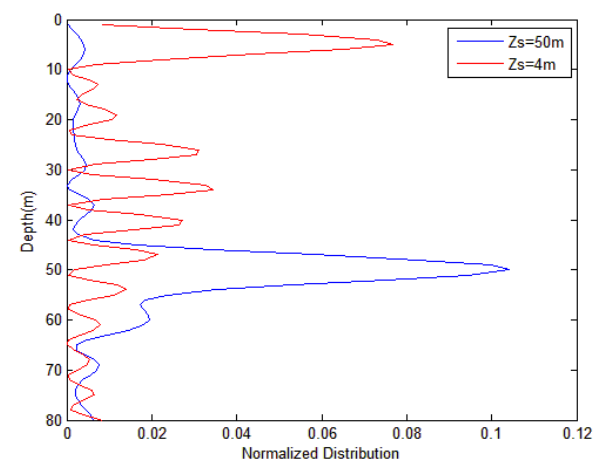

(d)

Figure 4. Comparison of $\mathrm{SAB}$ and MMAR in terms of source depth estimation by using simulated pressure field data covering the range span of $4990 \mathrm{~m}$ with an SNR of $40 \mathrm{~dB}$. (a) Mode wavenumber 
extracted using the modified Hankel transform of SAB. (b) Wavenumber estimated by the AR model of MMAR. (c) Depth estimation using SAB. (d) Depth estimation using MMAR.

The above mentioned analysis is based on the qualitative analysis of a single experiment, and the following two types of algorithms are analyzed quantitatively from the statistical point of view. In quantifying the estimated performance in an experiment, the correct index (CI) and output peak sidelobe ratio (OPSLR) should be defined. CI can be expressed as

$$
C I=\left\{\begin{array}{ll}
1 & \left|z_{e}-z_{s}\right| \leq g \\
0 & \left|z_{e}-z_{s}\right|>g
\end{array},\right.
$$

where $z_{e}$ is the result of source depth estimation and $g$ is the tolerance of the depth estimation. The OPSLR is defined as follows

$$
\text { OPSLR }=\left\{\begin{array}{cl}
\max (P(z))-\max \left(P\left(z^{\prime}\right)\right) & C I=1 \\
0 & C I=0
\end{array}\right.
$$

where

$$
\begin{gathered}
P(z)=20 \lg \frac{D(z)}{\max (D(z))}, \\
z \subseteq\left[z_{\min }, z_{\max }\right], \\
z^{\prime} \subseteq\left[z_{\min }, z_{s}-g\right] \cup\left[z_{s}+g, z_{\max }\right],
\end{gathered}
$$

We adopt the condition in Figure 4 and consider only the shallow source to intuitively understand the above-mentioned definition. The depth estimation results of the two algorithms are shown in Figure 5a. In this case, the two methods can estimate the source depth correctly. The OPSLR is marked for the two methods in Figure $5 \mathrm{~b}$. The OPSLR of SAB is $2.883 \mathrm{~dB}$ and that of MMAR is $10.34 \mathrm{~dB}$.

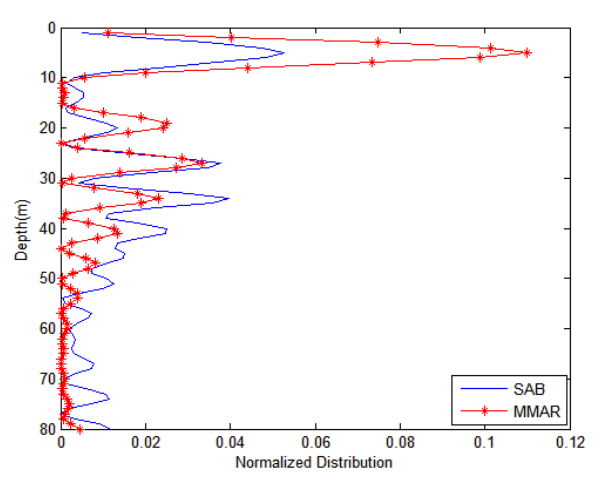

(a)

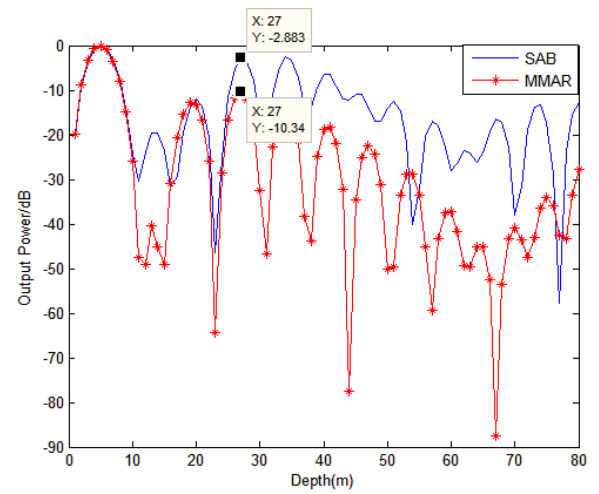

(b)

Figure 5. Depth estimation. (a) Normalized depth ambiguity functions, (b) Output power at all depths.

The correct probability (CP) and average peak sidelobe ratio (APSLR) are proposed to evaluate the statistical performance of source depth estimation in multiple experiments. CP can be expressed as $C P=\frac{1}{N_{0}} \sum_{n=1}^{N_{0}} C I$, and the APSLR is defined as APSLR $=\frac{1}{N_{0}} \sum_{n=1}^{N_{0}} O P S L R$, where is the 
281 found to better than the performance of that by SAB regardless of the source depth. The source

282

283

284

285

286

287 depth influences the algorithm as shown in Table 1 and 2. For effective comparison of performance of two algorithms, the source depth is varied from $1 \mathrm{~m}$ to $80 \mathrm{~m}$ in Figure 6 . The source moves $3990 \mathrm{~m}$ and SNR is $20 \mathrm{~dB}$. The similar regulation shown in Figure 6 can be found in other cases shown in Tables 1 and 2. Figure 6 shows that MMAR presents an improvement in depth estimation for different source depths compared with SAB.

Table 1. The results for the source depth of $4 \mathrm{~m}$.

\begin{tabular}{|c|c|c|c|c|c|}
\hline \multirow{2}{*}{ SNR(dB) } & \multirow{2}{*}{ Range(m) } & \multicolumn{2}{|c|}{$\mathrm{CP}$} & \multicolumn{2}{|c|}{ APSLR(dB) } \\
\hline & & SAB & MMAR & SAB & MMAR \\
\hline 40 & \multirow{4}{*}{1990} & 0.772 & 1.000 & 1.440 & 10.737 \\
\hline 30 & & 0.598 & 1.000 & 1.121 & 9.788 \\
\hline 20 & & 0.338 & 0.998 & 1.018 & 8.201 \\
\hline 15 & & 0.260 & 0.898 & 0.841 & 6.856 \\
\hline 20 & \multirow{2}{*}{2990} & 0.608 & 1.000 & 5.199 & 10.027 \\
\hline 10 & & 0.316 & 0.832 & 1.246 & 5.962 \\
\hline 20 & \multirow{2}{*}{3990} & 0.934 & 1.000 & 7.924 & 10.804 \\
\hline 10 & & 0.422 & 0.942 & 1.604 & 8.012 \\
\hline 10 & 4990 & 0.458 & 0.970 & 2.142 & 9.200 \\
\hline
\end{tabular}

Table 2. The results for the source depth of $50 \mathrm{~m}$.

\begin{tabular}{|c|c|c|c|c|c|}
\hline \multirow{2}{*}{$\mathrm{SNR}(\mathrm{dB})$} & \multirow{2}{*}{ Range(m) } & \multicolumn{2}{|c|}{$\mathrm{CP}$} & \multicolumn{2}{|c|}{ ASPLR(dB) } \\
\hline & & SAB & MMAR & SAB & MMAR \\
\hline 40 & \multirow{4}{*}{1990} & 0.000 & 1.000 & 0.000 & 25.907 \\
\hline 30 & & 0.000 & 1.000 & 0.000 & 20.491 \\
\hline 20 & & 0.000 & 1.000 & 0.000 & 20.788 \\
\hline 15 & & 0.002 & 1.000 & 0.007 & 19.192 \\
\hline 20 & \multirow{2}{*}{2990} & 0.670 & 1.000 & 2.345 & 24.510 \\
\hline 10 & & 0.484 & 1.000 & 2.602 & 19.420 \\
\hline 20 & \multirow{2}{*}{3990} & 0.680 & 1.000 & 5.804 & 25.830 \\
\hline 10 & & 0.820 & 1.000 & 6.017 & 21.508 \\
\hline 10 & 4990 & 0.704 & 1.000 & 8.901 & 21.845 \\
\hline
\end{tabular}




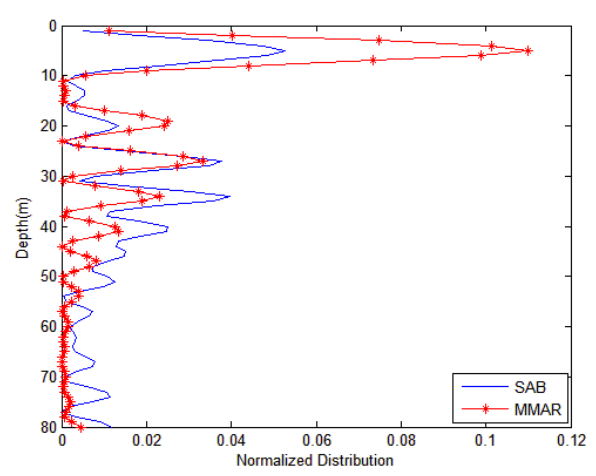

(a)

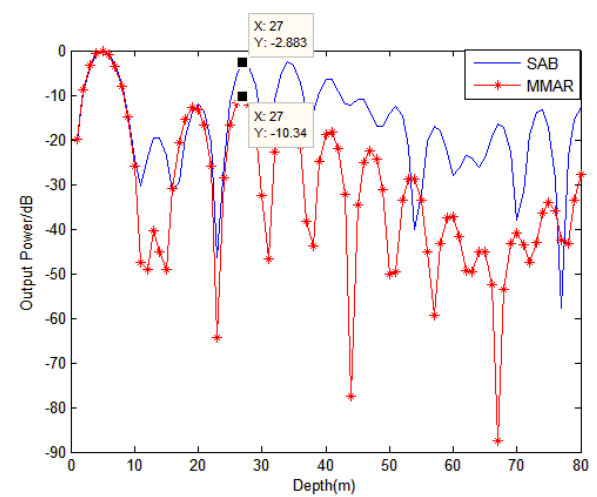

(b)

Figure. 6. Depth estimation performance for the various source depths by using (a) $\mathrm{CP}$ and (b) APSLR.

\subsection{Selection of the HLA depth}

After substituting Equation. (23) into Equation.(21), we can express the depth ambiguity function as

$$
D(z)=\left|\sum_{m=1}^{M} \phi_{m}(z) \frac{b_{m}}{1+\frac{\Delta^{2}}{\phi_{m}^{2}\left(z_{r}\right)}}\right|^{2}
$$

From Equation. (35), the mode is found to not contribute to depth estimation when the HLA depth $z_{r}$ coincides with the zero-crossing points of the mode depth function, that is, $\phi_{m}\left(z_{r}\right) \simeq 0$. The performance is influenced not only by the range span and the SNR, but also by the HLA depth.

In determining the influence of the HLA depth, we consider the two above-mentioned sources with a sufficient range span $(4990 \mathrm{~m})$ and high SNR. The HLA depth is varied from $1 \mathrm{~m}$ to $80 \mathrm{~m}$ to obtain the performance of depth estimation for different HLA depths. The number of Monte Carlo experiments is 500. Figure 7a shows the performance evaluated by CP with various HLA depths. For the deep source, the HLA depth significantly influences the correction of depth estimation. However, for the shallow source, CPs are all ones no matter where the HLA is located. This finding means that the HLA depth slightly influences the correctness of depth estimation. Consequently, as the source locates at different depths in the ocean, the HLA depth affects the source depth estimation differently. The performance evaluated by APSLR is shown in Figure $7 \mathrm{~b}$. For the deep source, when the HLA is located at $72 \mathrm{~m}$, the largest value of APSLR occurs. For the shallow source, the APSLR presents the largest value when the HLA depth is $36 \mathrm{~m}$. We can conclude from the above-mentioned observation that the most suitable HLA depth for depth estimation depends on the source depth. Thus, performance for various source depths and HLA depths should be studied. On the basis of the analysis present above, a suitable HLA depth span can be obtained for all source depths.

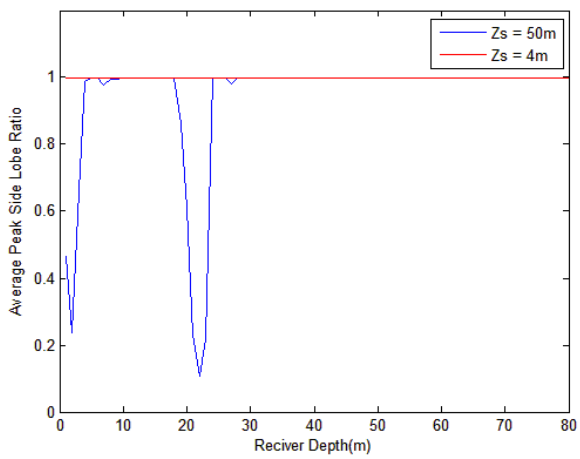

(a)

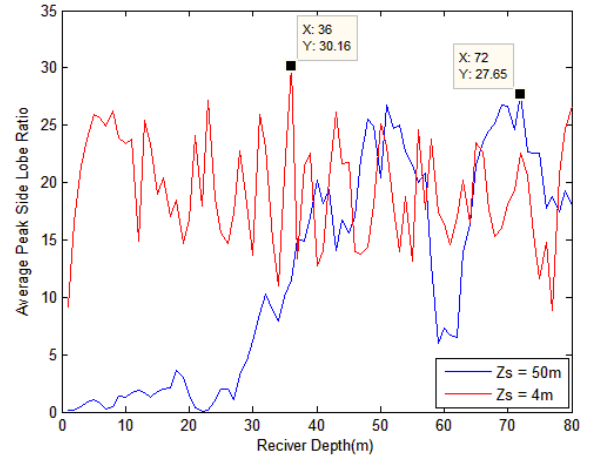

(b) 
Figure 7. Depth estimation performance for shallow and deep sources from a single receiver at various depth using (a) CP, and (b) APSLR.

A common conclusion for HLA depth span is obtained after evaluating the performance for all probable source depth. The performance evaluated by the APSLR for depth estimation under various source and HLA depths is shown in Figure 8. As shown in the figure, when the HLA is located near the water surface, the depth is difficult to be estimated correctly for some deep sources. The optimum placement depth of HLA differs for dissimilar source depths. The general trend is that, with the increasing of the source depth, the optimal placement depth of HLA also increases. In other words, the optimum placement depth of HLA is determined by the source depth. Therefore, we synthesize the depth estimation performance of the source with all possible depths and obtain the HLA depth span that is suitable for depth estimation. The distribution of the source depth is expressed by $p(z)$, and is assumed to be uniform. The result of the integrated correct estimation probability (ICP) for different HLA depths is present in Figure 9. The figure shows that, when HLA is located below $25 \mathrm{~m}$, the correctness of depth estimation can be guaranteed for all possible source depths. The integrated average peak sidelobe ratio (IAPSLR) as the placement depth of HLA increases is shown in Figure 9b. IAPSLR can be calculated by $p(z) \cdot A P S L R$. Synthesizing all possible source depths shows that, when the HLA depth is smaller than $30 \mathrm{~m}$, the IAPSLR increases with the placement depth of HLA. The general trend of the curve increases from $30 \mathrm{~m}$ to near the boundaries, with an oscillatory plateau in the local part. Furthermore, the IAPSLR presents higher value at this region than at the region of a source depth smaller than $30 \mathrm{~m}$. When the HLA is located at $72 \mathrm{~m}$, the IAPSLR possesses the largest value. These curves are valid for the given environment and frequency, but similar plateau can be observed with other downward-refracting SSPs. Consequently, when the HLA is located below the transitional layer, we can obtain a satisfying result of the source depth estimation. The conclusion is studied on the basis of the normal mode theory.

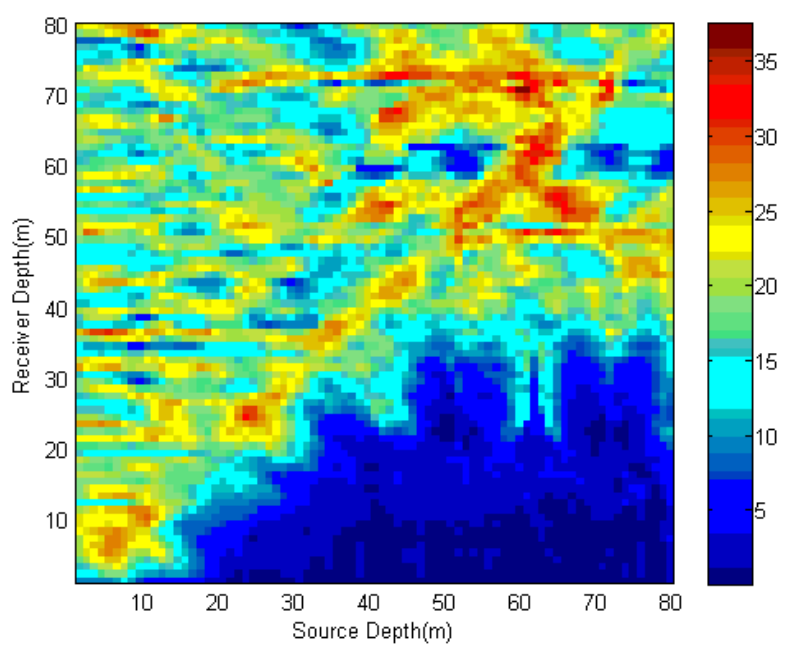

Figure 8. The performance of the depth estimation is evaluated by APSLR. The figure shows APSLR versus HLA and source depths. 


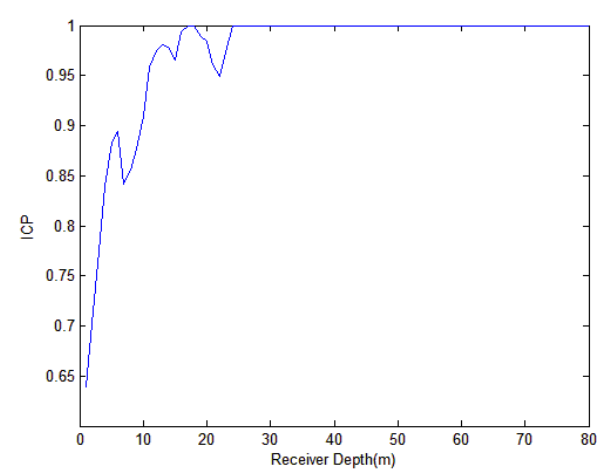

(a)

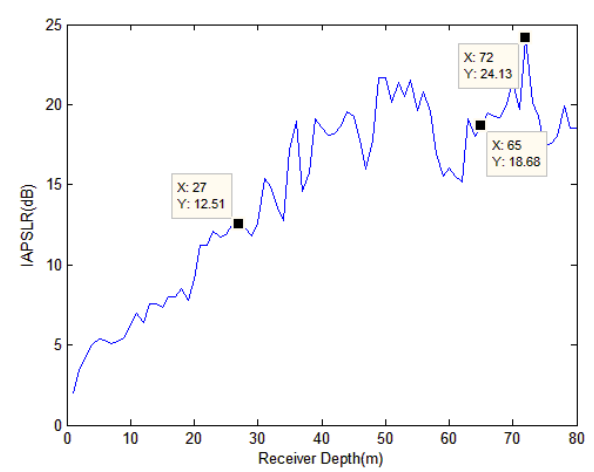

(b)

Figure 9. Curve for depth estimation performance with different HLA depths by using (a) ICP and (b) IAPSLR.

Figure 10 shows the 10 modes excited by the $350 \mathrm{~Hz}$ narrowband signal, which is radiated by the source located at $50 \mathrm{~m}$. The mode excitation amplitudes are the function of depth. For example, for the modes wherein the HLA is located at a range of $5010 \mathrm{~m}$ and a depth of $30 \mathrm{~m}$, several mode amplitudes (1th, 2nd, 8th, and 10th orders) is close to zero. The proposed method in this paper estimates the wavenumber before depth estimation. The wavenumbers are difficult to estimate when $\left|\phi_{m}\left(z_{r}\right) \phi_{m}\left(z_{s}\right)\right| \simeq 0$. The missing information degrades the performance of depth estimation.

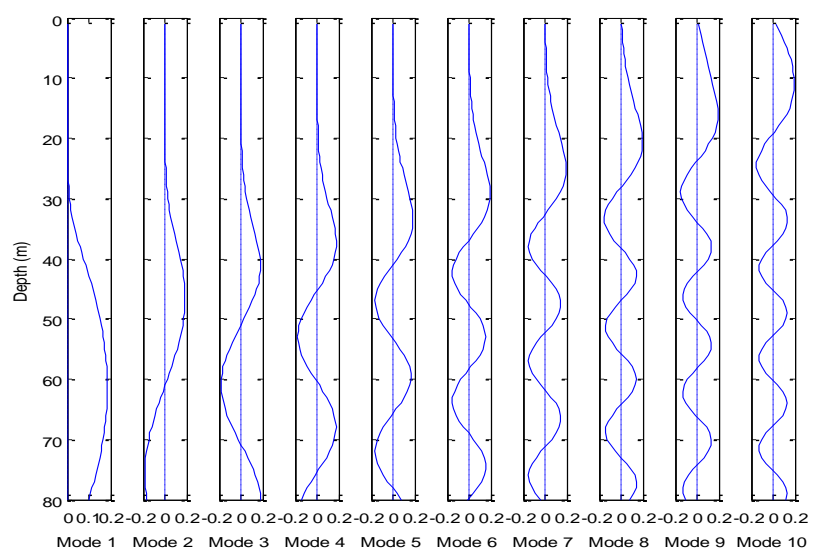

Figure 10. Ten order normal modes excited by the $350 \mathrm{~Hz}$ cw. Several mode amplitudes (1th, 2nd, 8 th, and 10th orders) is close to zero are shown in this figure.

We define the modes that contribute to the depth estimation as the modes with amplitudes larger than $\Delta / 2$. The number of modes that contribute to estimate source depth at various HLA depths is shown in Figure 11. Compared with other depths for HLA, $71 \mathrm{~m}$ and $72 \mathrm{~m}$ present a large number of the modes that contribute to depth estimation. This conclusion is the same as that for Figure $9 b$. In addition, the trend observed in Figure 11 is similar to that found in Figure 9b. 


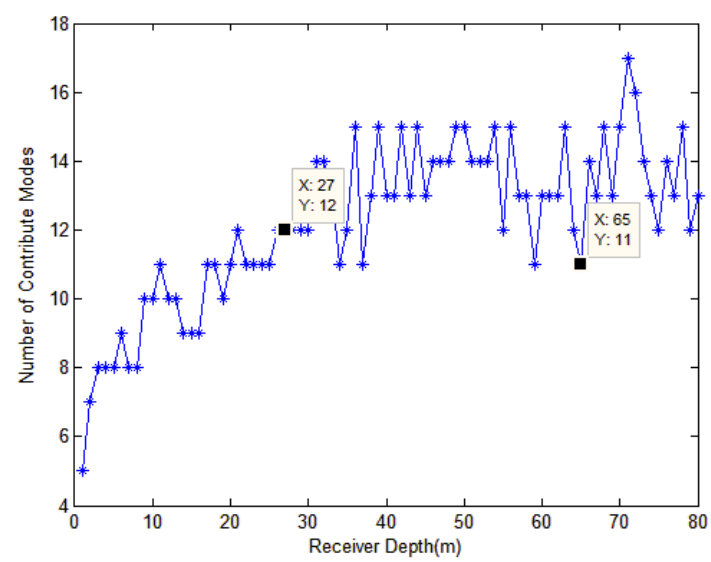

Figure 11. Number of modes that contribute to the source depth estimation at various receiver depths.

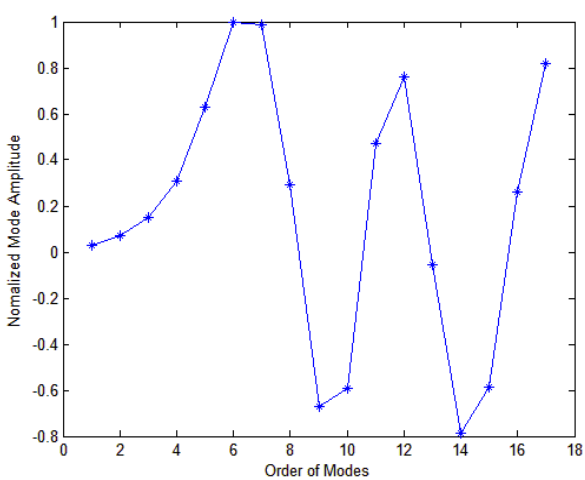

(a)

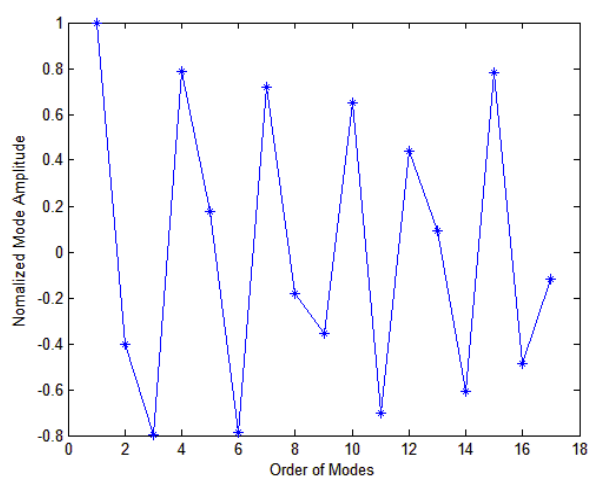

(b)

Figure 12. Variation curve of mode amplitudes and mode orders of modes when HLA is located at (a) $27 \mathrm{~m}$ and (b) $65 \mathrm{~m}$. 


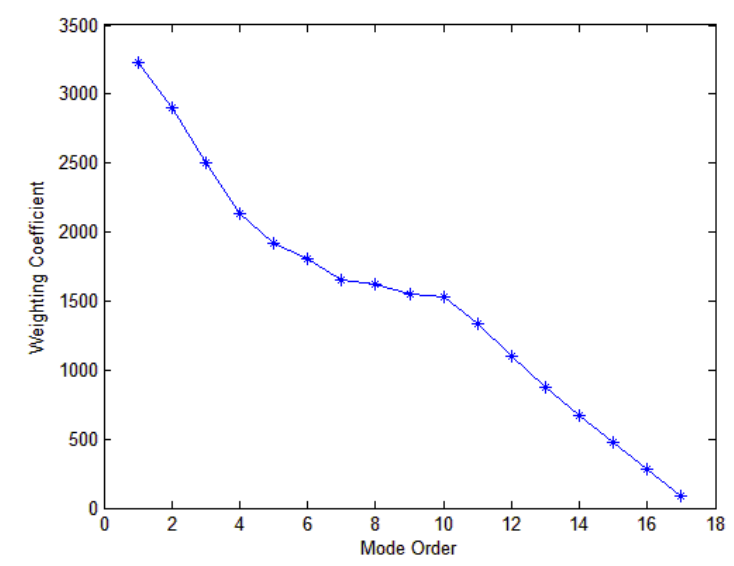

Figure. 13. Weighting coefficients decrease with the increasing mode order.

On the basis of the discussion presented above, the performance of depth estimation is determined by the number and the amplitudes of contributing modes, which are related with the HLA depth. The combined contribution of both to depth estimation can be expressed as follows

$$
\begin{gathered}
B=\sum_{m=1}^{M} c_{m} X_{m}, \\
X_{m}= \begin{cases}1 & \phi_{m}\left(z_{r}\right) \geq \Delta / 2 \\
0 & \phi_{m}\left(z_{r}\right)<\Delta / 2^{\prime}\end{cases}
\end{gathered}
$$

Figure 14 shows the contribution to depth estimation. Compared with Figure 11 and Figure 9b, a higher similarity is obtained between Figure 14 and Figure 9b. In summary, when the HLA is located below the transitional layer, the number of contributing modes is large and the lower order modes are dominant. Therefore, the combined contribution is larger in this case. Thus, a good depth estimation can be obtained when the HLA is located below the transitional layer.

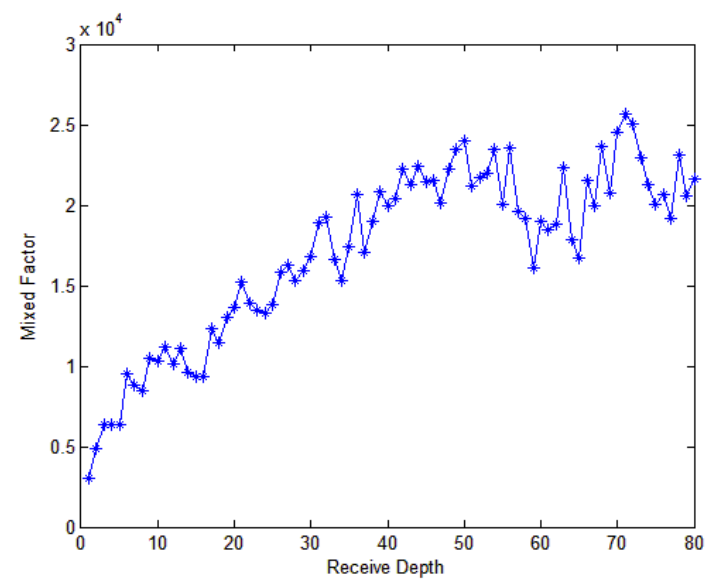

Figure. 14. Contribution to depth estimation.

\section{Conclusions}

A matched-mode method based on AR using an HLA to estimate the moving source depth is developed in this study. The modal wavenumber spectrum is obtained using generalized Hankel transform with the data from the moving source. The mode amplitudes can be extracted by combining the information based on AR modal wavenumber spectrum and the FT wavenumber spectrum. The amplitudes contain the information of source depth. The source depth is estimated by 
matching the mode estimation with the mode depth function calculated by the KRAKEN. The method is robust for estimating source depth as a result of the robustness of its mode depth function. The method proposed in this study is evaluated using the simulated data. For the data with a small range span or low SNR, the proposed method can achieve source depth estimation with better performance than SAB. The depth estimation performance is influenced not only by the range span and SNR but also by the HLA depth. The selection for HLA depth is studied and the result is explained by normal mode theory. In summary, when the HLA is located below the transitional layer, good depth estimation result can be expected. This conclusion is valid for the shallow waveguides with downward-refracting SSP.

Compared with that in $\mathrm{SAB}$, the requirement of the moving source traveling range decreases in proposed method. The proposed method can also be applied to the data with low SNR. However, this method is limited because it assumes the source depth fixed during the observation time. The effectiveness of this method degrades sharply, when the source depth varies rapidly. Thus, additional work is needed for its future application in source depth tracking.

Acknowledgments: This work was supported by the National Key R\&D Plan (2017YFC0306900), the National Natural Science Foundation of China ( 11504064 and 61405041 ), the Technology of Basic Scientific Research Project ( JSJL2016604B003 ), and the Open Fund for the National Laboratory for Marine Science and Technology of QingDao.

Author Contributions: Zhang Yi-Feng and Liang Guo-Long conceived and designed the experiments; Zhang Yi-Feng and Liang Guo-Long performed the experiments; Zhang Yi-Feng, Liang Guo-Long and Zou Nan analyzed the data; Wang Jin-Jin contributed reagents/materials/analysis tools; Zhang Yi-Feng wrote the paper.

Conflicts of Interest: The authors declare no conflict of interest. 
420

421

422

423

424

425

426

427

428

429

430

431

432

433

434

435

436

437

438

439

440

441

442

443

444

445

446

447

448

449

450

451

452

453

454

455

456

457

458

459

\section{References}

1. Baggeroer, A. B.; Kuperman, W. A.; Mikhalevsky, P. N. An overview of matched field methods in ocean acoustics. IEEE J. Ocean. Eng. 1993, 18 (4), 401-424, doi: 10.1109/48.262292.

2. Wang, Q; Wang, Y. M.; Zhu G. L. Matched field processing based on least squares with a small aperture hydrophone array. Sensors 2017, 17, 71, doi: 10.3390/s17010071.

3. Yang, T. C., A method of range and depth estimation by modal decomposition. The J. Acoust. Soc. Am. 1987, 82 (5), 1736-1745, doi: 10.1121/1.395825.

4. Gall, Y. L.; Socheleau, F. X.; Bonnel, J., Matched-Field Processing Performance Under the Stochastic and Deterministic Signal Models. IEEE Trans. Signal Process. 2014, 62 (22), 5825-5838, doi: 10.1109/TSP.2014.2360818.

5. Jesus, S. M.; Porter, M. B.; Stephan, Y.; Demoulin, X.; Rodriguez, O. C.; Coelho, E. M. M. F., Single hydrophone source localization. IEEE J. Ocean. Eng. 2002, 25 (3), 337-346, doi: 10.1109/48.855379.

6. Suppappola, S. B.; Harrison, B. F., Experimental matched-field localization results using a short vertical array and mid-frequency signals in shallow water. IEEE J. Ocean. Eng. 2004, 29 (2), 511-523, doi: 10.1109/JOE.2004.826896.

7. Yang, T. C., Source depth estimation based on synthetic aperture beamfoming for a moving source. J. Acoust. Soc. Am. 2015, 138 (3), 1678-1686, doi: 10.1121/1.4929748.

8. Rui, D.; Kun-De, Y.; Yuan-Liang, M.; Bo, L., A reliable acoustic path: Physical properties and a source localization method. Chin. Phys. B 2012, 21 (12), 276-289, doi: 10.1088/1674-1056/21/12/124301.

9. O., B. N.; A., B. P.; A., R. J.; W., S. P.; S., H. W.; L., D. S. G.; J., M. J., Source localization with broad-band matched-field processing in shallow water. IEEE J. Ocean. Eng. 1996, 21, 402, doi: 10.1109/48.544051.

10. Forero, P. A.; Baxley, P. A.; Straatemeier, L., A Multitask Learning Framework for Broadband Source-Location Mapping Using Passive Sonar. IEEE Trans. Signal Process. 2015, 63 (14), 3599-3614, doi: 10.1109/TSP.2015.2432747.

11. Bonnel, J.; Caporale, S.; Thode, A., Waveguide mode amplitude estimation using warping and phase compensation. J. Acoust. Soc. Am. 2017, 141 (3), 2243-2255, doi: 10.1121/1.4979057.

12. Yang, T. C.; Xu W., Data-based depth estimation of an incoming autonomous underwater vehicle. $J$. Acoust. Soc. Am. 2016, 140 (4), EL302-EL306, doi: 10.1121/1.4964640.

13. Yang, T. C., Data-based matched-mode source localization for a moving source. J. Acoust. Soc. Am. 2014, 135 (3), 1218-1230, doi: 10.1121/1.4863270.

14. Liu, H. W; Li, B. Q.; Yuan, X. B.; Zhou, Q. W.; Huang, J. C., A robust real time direction-of-arrival estimation method for sequential movement events of vehicles. Sensors 2018, 18, 992, doi:10.3390/s18040992.

15. Frisk, G. V.; Lynch, J. F., Shallow water waveguide characterization using the Hankel transform. J. Acoust. Soc. Am. 1984, 76 (1), 205-216, doi: 10.1121/1.391098.

16. Sarkar, T. K.; Pereira, O., Using the matrix pencil method to estimate the parameters of a sum of complex exponentials. IEEE Antennas Propag. Mag. 1995, 37 (1), 48-55, doi: 10.1109/74.370583.

17. Roux, P.; Cassereau, D.; Roux, A., A high-resolution algorithm for wave number estimation using holographic array processing. J. Acoust. Soc. Am. 2004, 115 (3), 1059-1067, doi: 10.1121/1.1648321. 